\title{
Serge GENEST
}

Anthropologue, professeur au département d’anthropologie, Université Laval

(1995)

\section{"L'engagement de Marc-Adélard Tremblay à l'enracinement de l'anthropologie au Québec”}

\author{
Un document produit en version numérique par Jean-Marie Tremblay, bénévole, \\ professeur de sociologie au Cégep de Chicoutimi \\ Courriel: jean-marie tremblay@uqac.ca \\ Site web pédagogique : http://www.uqac.ca/jmt-sociologue/ \\ Dans le cadre de: "Les classiques des sciences sociales" \\ Une bibliothèque numérique fondée et dirigée par Jean-Marie Tremblay, \\ professeur de sociologie au Cégep de Chicoutimi \\ Site web: http://classiques.uqac.ca/
}

Une collection développée en collaboration avec la Bibliothèque Paul-Émile-Boulet de l'Université du Québec à Chicoutimi Site web: http://bibliotheque.uqac.ca/ 


\section{Politique d'utilisation de la bibliothèque des Classiques}

Toute reproduction et rediffusion de nos fichiers est interdite, même avec la mention de leur provenance, sans l'autorisation formelle, écrite, du fondateur des Classiques des sciences sociales, Jean-Marie Tremblay, sociologue.

Les fichier des Classiques des sciences sociales ne peuvent sans autorisation formelle:

- être hébergé (en fichier ou page web, en totalité ou en partie) sur un serveur autre que celui des Classiques.

- servir de base de travail à un autre fichier modifié ensuite par tout autre moyen (couleur, police, mise en page, extraits, support, etc...),

Les fichiers (.html, .doc, .pdf., .rtf, .jpg, .gif) disponibles sur le site Les Classiques des sciences sociales sont la propriété des Classiques des sciences sociales, un organisme à but non lucratif composé exclusivement de bénévoles.

Ils sont disponibles pour une utilisation intellectuelle et personnelle et, en aucun cas, commerciale. Toute utilisation à des fins commerciales des fichiers sur ce site est strictement interdite et toute rediffusion est également strictement interdite.

L'accès à notre travail est libre et gratuit à tous les utilisateurs. C'est notre mission.

Jean-Marie Tremblay, sociologue

Fondateur et Président-directeur général, LES CLASSIQUES DES SCIENCES SOCIALES. 
Cette édition électronique a été réalisée par Jean-Marie Tremblay, bénévole, professeur de sociologie au Cégep de Chicoutimi à partir de :

Serge Genest

Anthropologue, professeur au département d'anthropologie, Université Laval.

"L'engagement de Marc-Adélard Tremblay à l'enracinement de l'anthropologie au Québec”.

Un article publié dans l’ouvrage sous la direction de François Trudel, Paul Charest et Yvan Breton, La construction de l'anthropologie québécoise. Mélanges offerts à Marc-Adélard Tremblay. Chapitre 31, pp. 457-459. Québec : Les Presses de l’Université Laval, 1995, 472 pp.

[Autorisation formelle accordée le 5 septembre 2007 par l'auteur de diffuser ce livre dans Les Classiques des sciences sociales.]

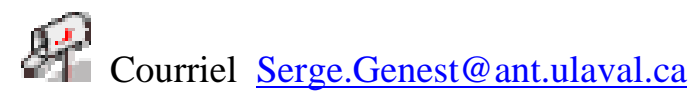

Polices de caractères utilisée :

Pour le texte: Times New Roman, 14 points.

Pour les citations : Times New Roman, 12 points.

Pour les notes de bas de page : Times New Roman, 12 points.

Édition électronique réalisée avec le traitement de textes Microsoft Word 2004 pour Macintosh.

Mise en page sur papier format : LETTRE (US letter), 8.5’’ x 11'’)

Édition numérique réalisée le 29 novembre 2007 à Chicoutimi, Ville de Saguenay, province de Québec, Canada.

\section{Fait avec}

Macintosh 


\section{Serge Genest}

Anthropologue, professeur au département d'anthropologie,

Université Laval.

\section{"L'engagement de Marc-Adélard Tremblay à l'enracinement de l'anthropologie au Québec”}

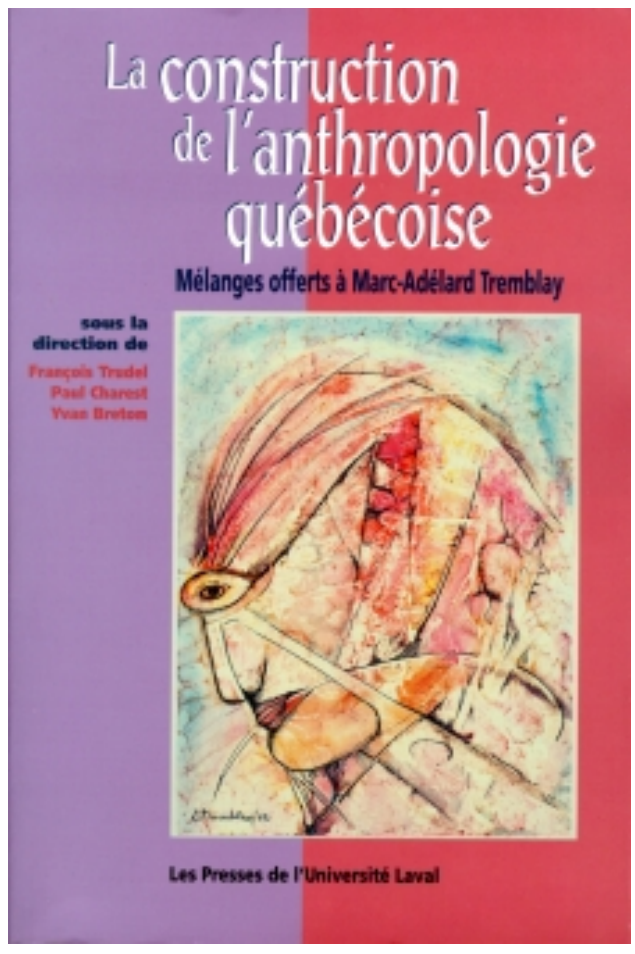

Un article publié dans l'ouvrage sous la direction de François Trudel, Paul Charest et Yvan Breton, La construction de l'anthropologie québécoise. Mélanges offerts à Marc-Adélard Tremblay. Chapitre 31, pp. 457-459. Québec : Les Presses de l’Université Laval, 1995, 472 pp. 


\section{Serge Genest \\ Anthropologue, professeur au département d'anthropologie, Université Laval. \\ “L'engagement de Marc-Adélard Tremblay \\ à l'enracinement de l'anthropologie au Québec”.}

Un article publié dans l'ouvrage sous la direction de François Trudel, Paul Charest et Yvan Breton, La construction de l'anthropologie québécoise. Mélanges offerts à Marc-Adélard Tremblay. Chapitre 31, pp. 457-459. Québec: Les Presses de l’Université Laval, 1995, 472 pp.

Il suffit de fréquenter les universitaires durant un court laps de temps pour constater combien le développement de la carrière professionnelle personnelle revêt d'importance. Comme une hantise qu'on ignore leur existence. Et personne n'échappe vraiment à cette tendance.

Il faut dire que les structures de travail dans lesquelles évoluent les universitaires, le "publie-ou-meurt », conditionnent en partie pareil comportement. Peut-être aussi de nombreuses années d'effort à se construire une identité dans un monde de compétition sans âme.

À considérer la production intellectuelle et le cheminement de carrière de Marc-Adélard Tremblay, on pourrait sans difficulté y voir un autre exemple de cette crainte de sombrer dans l'oubli ou de la volonté de se forger une identité à tout prix. Pourtant, ce ne sont pas de ces motivations dont je veux parler ici. J'entends plutôt témoigner de celles, bien différentes, de quelques individus qui fréquentent d'autres sentiers durant leur vie d'universitaire, qui se font un devoir d'aller audelà de leur ambition personnelle. C'est cet esprit que j'ai trouvé chez Marc-Adélard Tremblay depuis mes premiers contacts avec lui, il y a maintenant quelque vingt-cinq ans. 
Il peut paraître prétentieux que le cadet que je suis s'adresse publiquement à celui qui fut son professeur. Les lignes qui suivent se veulent cependant une reconnaissance toute simple de ce que moi et tant d'autres devons à la volonté de Marc-Adélard Tremblay d'inscrire l'anthropologie dans la société québécoise contemporaine. Je crois aussi que toutes ces années à le côtoyer comme étudiant, jeune chercheur et collègue de travail, me donnent quelque autorité pour faire état de l'engagement que j'ai constaté chez lui envers les jeunes générations d'anthropologues.

Ma toute première expérience de l'attention portée par MarcAdélard aux anthropologues en formation remonte à l'époque où, jeune étudiant au milieu d'une scolarité de baccalauréat en anthropologie, j'ai profité de ses conseils et de son appui pour obtenir une bourse d'études d'une fondation américaine. Grâce à ses contacts, il avait su faire comprendre l'importance et l'intérêt, pour ce programme de bourses, d'aider de jeunes anthropologues francophones.

Depuis, combien d'autres personnes ont pu ainsi bénéficier de ce même soutien? Sans doute plusieurs, car jusqu'à aujourd'hui MarcAdélard s'est fait un devoir de soutenir les candidatures jugées prometteuses. Il ne s'agit pas seulement d'appuis de " circonstance », dirais-je. C'est véritablement un engagement pour que ces personnes trouvent les moyens financiers de développer leurs aptitudes à la recherche. Sans qu'il n'y paraisse, ce travail exige qu'on mette le temps nécessaire à fournir pour elles les évaluations pertinentes et aussi qu'on ait développé un bon réseau d'information et de contacts pour faire valoir leurs candidatures.

Ce travail précieux et gratuit de repérage de futurs anthropologues ne peut s'effectuer qu'à partir d'une conviction profonde de la continuité dans le développement de l'anthropologie. 
Cette attitude transparaît aussi dans l'attention portée à la formation à la recherche. Malgré le temps nécessairement passé à sa propre production scientifique, Marc-Adélard a aussi voulu s'assurer que des projets soient mis en place pour que des étudiantes et des étudiants trouvent l'occasion de faire du terrain. Il est à l'origine d'une vaste entreprise, échelonnée sur près de dix ans, le « Projet Côte-Nord », qui fut l'occasion pour moi et pour toute une génération d'anthropologues de l'Université Laval de se former à la recherche sous la supervision de personnes plus expérimentées.

Dans ce type d'entreprise, non seulement il faut consacrer du temps et des énergies à développer les projets et à obtenir des subventions, autrement dit à construire les environnements intellectuel et logistique appropriés, mais il faut en plus, je crois, une vision très profonde de l'importance de ce travail. Marc-Adélard a transmis à plusieurs sa façon d'envisager la recherche, mais une telle vision n'est pas encore monnaie courante. Il demeure plus tentant de se concentrer sur ses seules productions. Marc-Adélard a choisi une autre voie, celle de l'investissement dans l'anthropologie de demain. Il le fait encore maintenant, à l'heure même où il s'apprête à quitter la vie universitaire.

Comme étudiant, comme jeune chercheur, j'ai personnellement bénéficié des actions concrètes accomplies par Marc-Adélard pour promouvoir le développement de l'anthropologie. Mais c'est en tant que collègue que j'ai pu saisir toute la qualité de son engagement à assurer une relève compétente chez les anthropologues du Québec.

Depuis quelques années, en effet, je participe, au gré des demandes des personnes inscrites dans nos programmes de maîtrise et de doctorat, à des comités de supervision en compagnie de Marc-Adélard. Nos compétences respectives en matière d'anthropologie médicale nous ont permis de travailler régulièrement ensemble dans ce contexte. C'est au fil de ces rencontres de travail que j'ai pu encore davantage apprécier les efforts qu'il déploie à l'endroit des nouvelles générations d'anthropologues. 
Combien de fois ne l'ai-je pas vu, avec application et rigueur, replacer une étudiante ou un étudiant sur ses rails dans l'élaboration d'une problématique de recherche un peu laborieuse ! Toujours avec le même souci d'aider et de faire progresser le travail dans le respect des idées des personnes intéressées. Après tant d'années passées à ce type d'activité, on pourrait s'attendre à de la lassitude, voire à un certain relâchement. Pourtant, c'est de l'enthousiasme, de l'intérêt pour les personnes qu'affiche Marc-Adélard. J'avoue trouver beaucoup de stimulation et de fraîcheur dans cette attitude. J'ai puisé là des énergies bénéfiques et un enseignement sur l'engagement envers les futurs anthropologues.

Et comme si cela ne suffisait pas, Marc-Adélard trouve encore le moyen, lorsqu'on lui en fait la demande ou qu'il juge lui-même des qualités d'une personne, de mettre en action les réseaux qu'il a construits dans des milieux très divers pour aider au démarrage d'une carrière d'anthropologue. Je revois ainsi se dérouler devant moi la même pratique qui m'a permis, il y a déjà plus de 25 ans, de forger mes premières armes de chercheur.

L'enseignement que je retiens personnellement de mes contacts avec Marc-Adélard Tremblay est celui d'un engagement indéfectible au progrès de l'anthropologie au Québec. Il faut, à mon avis, une vision claire d'enracinement de l'anthropologie pour avoir pu, au long de tant d'années d'enseignement et de recherche, garder le même cap : fournir les meilleures conditions intellectuelles et matérielles aux générations montantes d'anthropologues et travailler à la diffusion de l'anthropologie dans des milieux de recherche fondamentale autant que d'application des connaissances.

C'est un très grand plaisir pour moi de rendre hommage à MarcAdélard Tremblay comme ancien étudiant, comme chercheur et comme collègue. 